\title{
Acute Onset Diplopia and Squint as the Only Presentation of Idiopathic Intracranial Hypertension in a Six-Year-Old
}

\author{
Sohan Lohiya ${ }^{1}$, Pravin Tidake², Ruta Walavalkar ${ }^{3}$ \\ 1, 2,-3 Department of Ophthalmology, Jawaharlal Nehru Medical College, Datta Meghe Institute of Medical Sciences \\ (Deemed to Be University), Sawangi, Meghe, Wardha, Maharashtra, India.
}

\section{INTRODUCTION}

Idiopathic intracranial hypertension (IIH), also referred to as pseudotumour cerebri, is characterized by elevated intracranial pressure without discernible aetiology, with normal cerebrospinal fluid content, and normal contrast-enhanced computerised tomography (CT) or magnetic resonance imaging. Pseudotumour cerebri has been well described in adults, where there is a strong female preponderance and an association with obesity. It is relatively uncommon in children and adolescents and in most cases may be associated with puberty and the resulting hormonal changes. Prepubertal children usually present with stiff neck, diplopia and strabismus.

This is a case report of a 6-year-old female presenting with acute onset diplopia and lateral rectus paralysis in right eye to Ophthalmology OPD. On doing a thorough clinical examination, the child was referred to paediatrics department with a strong suspicion of idiopathic intracranial hypertension. The neuro-paediatrician started the child on anti-oedema measures. Magnetic resonance venography suggested mild distension of sagittal sinus thus confirming the diagnosis.

Though idiopathic intracranial hypertension is extremely rare in childhood, its possibility has to be ruled out when presented with suggestive symptoms and signs. As early diagnosis and management can be very crucial in the prognosis and ocular development in such young individuals.

\section{PRESENTATION OF CASE}

A 6-year-old female presented to Ophthalmology OPD with sudden onset of double vision and deviation of right eye (Figure 1) since one day. Patient did not give history of headache, vomiting, fever, seizure, walking difficulty, diminution of vision, and other ocular or systemic complaints. There was no history of trauma, previous surgery or systemic illness. Patient was first-born child of the parents with unremarkable pre-natal birth and post-natal history. Developmental milestones were achieved on time. Her immunization status was complete and up-to-date.

Central nervous system examination revealed active, alert and conscious patient with normal tone, power and reflexes both superficial and deep. No abnormality detected in cardiovascular, respiratory and gastrointestinal systems. On ocular examination, no epicanthal folds, mongoloid slant of palpebral fissure or hypertelorism. Hirschberg test showed 45-degree esotropia in right eye. Rest of anterior segment was within normal limit. Both pupils were normal in size and reacting to light. Extra-ocular movement examination revealed restricted abduction in right eye. Fundus glow through direct ophthalmoscope was normal and bright. Best corrected visual acuity was 6 / 9 in both eyes. Fundus examination revealed elevated disc margins in both eyes.
Corresponding Author: Dr. Ruta Walavalkar, F-9/Radhikabai Girl's Hostel, JNMC Campus, Sawangi, Meghe, Wardha, Maharashtra, India.

E-mail: rutawalavalkar@gmail.com

DOI: $10.14260 /$ jemds/2021/72

How to Cite This Article:

Lohiya S, Tidake P, Walavalkar R. Acute onset diplopia and squint as the only presentation of Idiopathic Intracranial Hypertension in a six year old. J Evolution Med Dent Sci 2021;10(05):323-325, DOI: 10.14260/jemds/2021/72

Submission 06-10-2020,

Peer Review 01-12-2020,

Acceptance 07-12-2020,

Published 01-02-2021.

Copyright (c) 2021 Sohan Lohiya et al. This is an open access article distributed under Creative Commons Attribution License [Attribution 4.0 International (CC BY 4.0)] 

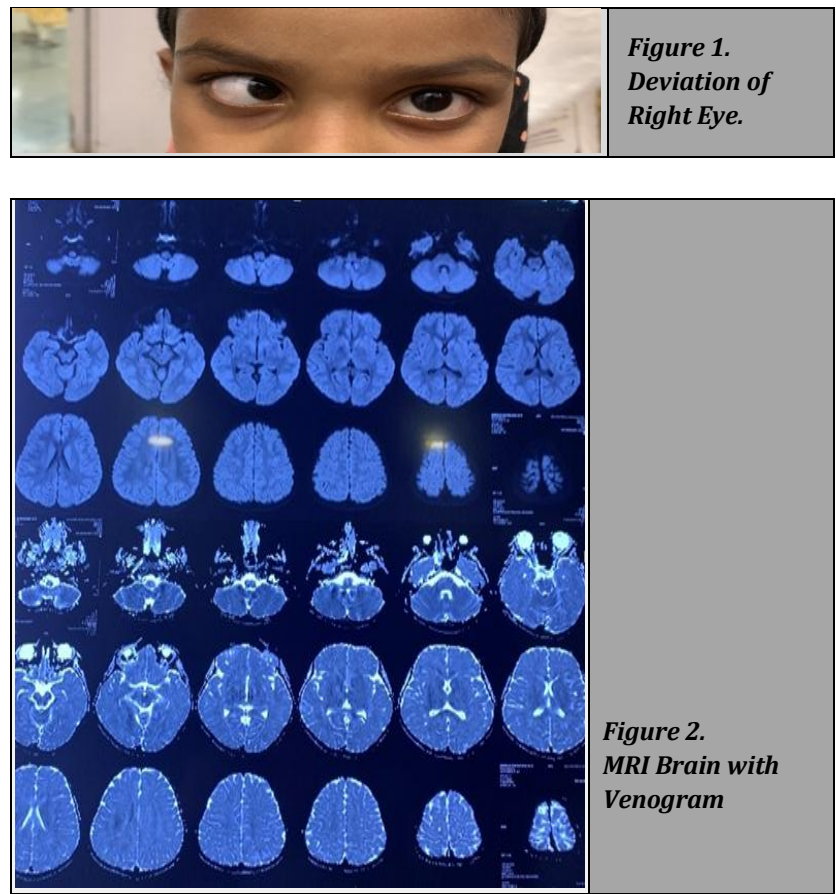

\section{CLINICAL DIAGNOSIS}

A provisional diagnosis of raised intracranial pressure was made and the patient was referred to paediatric neurology. The neurologist advised admission and complete blood and urine investigations. Haematological examination showed mild leukocytosis ( 12400 cells / cumm) while rest values were within normal limit. Magnetic resonance imaging (MRI) brain with venogram (Figure 2) was advised, MRI study of brain did not reveal any significant structural or parenchymal abnormality, while MR venography of brain revealed mild distension of superior sagittal sinus.

\section{DISCUSSION OF MANAGEMENT}

Patient was started on anti-oedema measures i.e., injection mannitol $40 \mathrm{~mL}$ stat followed by $20 \mathrm{~mL}$ three times a day (TDS) with blood pressure monitoring.

Indirect ophthalmoscopy was performed again after one week which revealed well defined margins of optic disc in both eyes thus ruling out papilloedema. Lumbar puncture was then done to study cerebrospinal fluid (CSF) microscopy and culture with CSF pressure monitoring. CSF examination was within normal limits except mild increase in glucose level (73 $\mathrm{mg} / \mathrm{dL}$ ref range- $40-70 \mathrm{mg} / \mathrm{dL}$ ). As the only pathology was found to be the mild distension of sagittal sinus on MR venogram a final diagnosis of idiopathic intracranial hypertension was made. Patient was then started on intravenous (IV) dexamethasone and oral acetazolamide (250 mg) 1 tab. TDS. Patient was discharged against medical advice within 2 days on request of parents on Tab. acetazolamide (250 mg) 1 tab. TDS, oral prednisolone tapering dose and calcium syrup and was advised follow up after 1 week.

Patient came to Ophthalmology OPD after 2 weeks of treatment, and still complained of diplopia and deviation of right eye. Her best corrected visual acuity was 6 / 9 in both eyes. Hirschberg test showed 45-degree esotropia in right eye though extra-ocular movements were mildly restricted in right eye during abduction as compared to before. Fundus examination showed no signs of papilloedema in both eyes. Visual field analysis was advised for the patient but could not be performed as patient was not co-operative. Patient was started on patch therapy. The rationale behind starting the patient on patch therapy was that as the strabismus has not been corrected, the patient might develop strabismic amblyopia and will lead to diminution of vision as the patient gets older. Parents were counselled and advised admission of the child for strabismus correction surgery after paediatric fitness.

\section{DISCUSSION}

Paediatric intracranial hypertension is rare. ${ }^{1} \mathrm{~A}$ review of the literature shows that most series have been collected at major paediatric referral centres over a period of 10 to 20 years and have included older children who may have already undergone puberty and the resulting hormonal changes. ${ }^{2}$ In prepubertal children, idiopathic intracranial hypertension appears to be even less frequent. One report found that $60 \%$ of children with idiopathic intracranial hypertension were older than 10 years. ${ }^{2-6}$ In studies where paediatric intracranial hypertension was studied, among the signs and symptoms which included strabismus, diplopia, papilloedema and visual field defects, the commonest presentation was that of diplopia and strabismus with $6^{\text {th }}$ cranial nerve palsy to be the most common among all other cranial nerve abnormalities, thus child presenting with lateral rectus palsy.

Although in majority cases, the diplopia and strabismus resolved after normalization of intracranial pressure, in our case report of a six-year-old female, resolution of lateral rectus palsy has not occurred even after a month of medical management.7,8 The associated papilloedema has, however, resolved and patient has reported decrease in the level of diplopia now as compared to that before the treatment.

The pit-falls of this case would be the inability to perform perimetry which would have suggested visual field defect if any, which is a very common finding in cases of pseudotumour cerebri. Furthermore, no significant resolution of the ocular symptoms even after prompt medical management has occurred.

Financial or other competing interests: None.

Disclosure forms provided by the authors are available with the full text of this article at jemds.com.

\section{REFERENCES}

[1] Jirásková N, Rozsíval P. Idiopathic intracranial hypertension in pediatric patients. Clin Ophthalmol 2008;2(4):723-6.

[2] Weisberg LA, Chutorian AM. Pseudotumor cerebri of childhood. Am J Dis Child 1977;131(11):1243-8.

[3] Baker RS, Baumann RJ, Buncic JR. Idiopathic intracranial hypertension (pseudotumor cerebri) in pediatric patients. Pediatr Neurol 1989;5(1):5-11. 
[4] Grant DN. Benign intracranial hypertension. A review of 79 cases in infancy and childhood. Arch Dis Child 1971;46(249):651-5.

[5] Dhiravibulya K, Ouvrier R, Johnston I, et al. Benign intracranial hypertension in childhood: a review of 23 patients. J Paediatr Child Health 1991;27(5):304-7.

[6] Hagberg B, Sillanpaa M. Benign intracranial hypertension (pseudotumor cerebri). Review and report of 18 cases. Acta Pediatr Scand 1970;59(3):328-9.
[7] Babikian P, Corbett J, Bell W. Idiopathic intracranial hypertension in children: the Iowa experience. J Child Neurol 1994;9(2):144-9.

[8] Weig SG. Asymptomatic idiopathic intracranial hypertension in young children. J Child Neurol 2002;17(3):239-41. 